\title{
POWERS OF THE VERTEX COVER IDEALS
}

\author{
FATEMEH MOHAMMADI
}

\begin{abstract}
We describe a combinatorial condition on a graph which guarantees that all powers of its vertex cover ideal are componentwise linear. Then motivated by Eagon and Reiner's Theorem we study whether all powers of the vertex cover ideal of a CohenMacaulay graph have linear free resolutions. After giving a complete characterization of Cohen-Macaulay cactus graphs (i.e., connected graphs in which each edge belongs to at most one cycle) we show that all powers of their vertex cover ideals have linear resolutions.
\end{abstract}

\section{INTRODUCTION}

Let $G$ be a graph on the vertex set $V(G)=\left\{x_{1}, \ldots, x_{n}\right\}$ with the edge set $E(G)$, and let $R=K\left[x_{1}, \ldots, x_{n}\right]$ be the polynomial ring over a field $K$. Two monomial ideals are associated to $G$, the edge ideal $I(G)$ generated by all monomials $x_{i} x_{j}$ with $\left\{x_{i}, x_{j}\right\} \in E(G)$, and the vertex cover ideal $J(G)$ generated by monomials $\prod_{x_{i} \in C} x_{i}$ for all minimal vertex covers $C$ of $G$. We recall that a minimal vertex cover of $G$ is a subset $C \subset V(G)$ such that each edge has at least one vertex in $C$ and no proper subset of $C$ has the same property. The vertex cover ideal $J(G)$ is the Alexander dual of the edge ideal of $G$, i.e. $J(G)=I(G)^{\vee}=\bigcap_{\left\{x_{i}, x_{j}\right\} \in E(G)}\left(x_{i}, x_{j}\right)$.

A homogeneous ideal $I \subset R$ is called componentwise linear if for each $d$, the ideal $\left(I_{d}\right)$ generated by all forms of degree $d$ in $I$ has a linear resolution, see [9]. A criteria for an ideal being componentwise linear is given in [1]. Francisco and Van Tuyl showed that the vertex cover ideal of a chordal graph is componentwise linear, and recently Herzog, Hibi and Ohsugi studied the powers of these ideals, and they conjectured that all powers of the vertex cover ideal of a chordal graph are componentwise linear, see [5, 10]. We are interested in the following question: Do there exist non-chordal graphs such that all powers of their vertex cover ideals are componentwise linear? This kind of problem is studied by Nevo and Peeva for the powers of some edge ideals, see [17, 18].

On the other hand, in general it is hard to prove that a class of ideals has linear resolutions, and even having an ideal with a linear resolution, does not guarantee to have the same property for its powers, see [20]. Therefore it is natural to study the problem for the vertex cover ideals of graphs, and investigate some combinatorial properties of graphs which are sufficient to have minimal linear resolutions for ideals $J(G)^{k}$. In this point of view, bipartite and chordal graphs have been studied in [8, 10]. A graph $G$ is called (sequentially) Cohen-Macaulay if the quotient ring $R / I(G)$ is (sequentially) CohenMacaulay over every field $K$. As a consequence of Eagon and Reiner's Theorem, we have

2010 Mathematics Subject Classification. 13C40, 13H10, 13P10, $05 \mathrm{E} 40$.

Key words and phrases. Cohen-Macaulay graphs, componentwise linear ideals, edge ideals, vertex cover ideals of graphs.

The author acknowledges support from the Mathematical Sciences Research Institute in Berkeley and the Alexander von Humboldt Foundation in Germany. 
that a graph is Cohen-Macaulay if and only if its vertex cover ideal has a linear resolution. One can raise this question: given an arbitrary Cohen-Macaulay graph, what can be said about the powers of its vertex cover ideal? Does each power of the vertex cover ideal of a Cohen-Macaulay graph have a linear resolution? As a consequence of [10, Theorem 3.7] we know that all powers of the vertex cover ideal of a Cohen-Macaulay chordal graph have linear resolutions. Also it is shown that all powers of the vertex cover ideal of a Cohen-Macaulay bipartite graph are componentwise linear, see [8]. In fact in Theorem 2.3 and some results of [14, 15] mentioned in the last part, it is shown that all powers of the vertex cover ideal a Cohen-Macaulay bipartite graph are weakly polymatroidal ideals and so they have linear resolutions.

As Herzog, Hibi and Zheng described in [11] classifying Cohen-Macaulay graphs in general is as hard as classifying all Cohen-Macaulay simplicial complexes. However finding some combinatorial conditions equivalent to Cohen-Macaulay property in graphs has been extensively studied by several authors in [4, 7, 11, 22]. The problem is solved for two well-known families of graphs, chordal and bipartite graphs, and it was shown that in these graphs the Cohen-Macaulay property is independent of the field $K$, see [7, 11].

The paper is structured as follows. In $\S 1$ we investigate a combinatorial condition involving the number of free vertices in the clique complex of $G$ that guarantees that all powers of $J(G)$ have linear quotients (and hence componentwise linear resolutions), see Theorem 1.3. In $\S 2$ we study the ideals arising from a cactus graph. We give a combinatorial characterization of Cohen-Macaulay cactus graphs, see Theorem 2.3, and as a consequence we see that in cactus graphs the Cohen-Macaulay property is independent of the field $K$. Using this characterization in $\S 3$ we show that all powers of the vertex cover ideal of a Cohen-Macaulay cactus graph are weakly polymatroidal and thus have linear resolutions, see Theorem 3.3 .

\section{COMPONENTWISE LINEAR VERTEX COVER IDEALS}

A vertex cover $C$ of $G$ is a subset of $V(G)$ which meets every edge of $G$. A minimal vertex cover of $G$ is a vertex cover $C$ that there is no subset $C^{\prime} \subset C$ such that $C^{\prime}$ is a vertex cover of $G$. The vertex cover ideal of $G$ is the Alexander dual of the edge ideal of $G, J(G)=I(G)^{\vee}=\bigcap_{\left\{x_{i}, x_{j}\right\} \in E}\left(x_{i}, x_{j}\right)$. For any monomial ideal $I$, we denote by $G(I)$ the minimal set of generators of $I$, and we denote by $\operatorname{deg}_{x_{i}} f$ the exponent of the variable $x_{i}$ in the monomial $f$. For two monomials $f$ and $g$ we say $f>_{l e x} g$ if and only if the left-most nonzero entry in the sequence $\left(\operatorname{deg}_{x_{1}} f-\operatorname{deg}_{x_{1}} g, \ldots, \operatorname{deg}_{x_{n}} f-\operatorname{deg}_{x_{n}} g\right)$ is positive. In the following by $>$, we mean $>_{\text {lex }}$.

We recall that the monomial ideal $I \subset R$ has linear quotients, if there exists a system of minimal generators $f_{1}, f_{2}, \ldots, f_{m}$ of $I$ such that the colon ideal $\left(f_{1}, \ldots, f_{i-1}\right): f_{i}$ is generated by a subset of $\left\{x_{1}, \ldots, x_{n}\right\}$ for all $i$. Ideals with linear quotients were introduced by Herzog and Takayama in [12. A class of ideals enjoying the nice property of having linear quotients is the class of weakly polymatroidal ideals introduced by Hibi and Kokubo in [13] for ideals generated in the same degree (and later in [15] for ideals not necessarily generated in one degree).

Definition 1.1. A monomial ideal $I \subset R$ is weakly polymatroidal if for any two monomials $f=x_{1}^{a_{1}} \cdots x_{n}^{a_{n}}$ and $g=x_{1}^{b_{1}} \cdots x_{n}^{b_{n}}$ in $G(I)$ with $a_{1}=b_{1}, \ldots, a_{t-1}=b_{t-1}$ and $a_{t}>b_{t}$, there exists $\ell>t$ such that $x_{t}\left(g / x_{\ell}\right) \in I$. 
For each simple graph $G$, the simplicial complex on $V(G)$ whose faces are the cliques of $G$ is called the clique complex of $G$ denoted by $\Delta(G)$. A vertex $v \in V(G)$ is a free vertex if it belongs just to one clique of $G$. We denote by $\operatorname{deg}_{G}(u)$ the number of the adjacent vertices to $u$ in $G$. For more background, we refer to [6, 19, 22] for the combinatorial point of view. We denote by $\mathcal{F}(\Delta(G))$ the set containing of all facets of $\Delta(G)$ with free vertices. The main result of this section, Theorem 1.3, explains having enough free vertices in a graph is sufficient to have componentwise linear vertex cover ideals. These graphs are not necessarily Cohen-Macaulay and they may not have linear resolutions, but they have componentwise linear vertex cover ideals.

The proof of componentwise linearity of these ideals depends on describing a nice labeling on the vertices of their corresponding graphs. Then we fix the natural lexicographic order on the variables corresponding to the vertices of $G$. This term order allows us to check the condition of weakly polymatoidals for our ideals which implies having linear quotients.

Example 1.2. The vertex cover ideal of the graph $P_{4}$ on the vertices $v_{1}, v_{2}, v_{3}, v_{4}$ with the edges $v_{1} v_{2}, v_{2} v_{3}, v_{3} v_{4}$ is weakly polymatroidal. We consider a new labeling on the vertices to have first non-free vertices $v_{2}, v_{3}$ and then free vertices $v_{1}, v_{4}$ of $P_{4}$. Then we set $x_{1}:=v_{2}, x_{2}:=v_{3}, x_{3}:=v_{1}$ and $x_{4}:=v_{4}$. The edges of $P_{4}$ are $x_{3} x_{1}, x_{1} x_{2}, x_{2} x_{4}$, and the vertex cover ideal is $J\left(P_{4}\right)=\left(x_{1} x_{2}, x_{1} x_{4}, x_{2} x_{3}\right)$. Now an easy computation shows that $J\left(P_{4}\right)$ is weakly polymatroidal.

Theorem 1.3. Let $G$ be a graph with $\left|\bigcup_{F \in \mathcal{F}(\Delta(G))} F\right| \geq|V(G)|-1$. Then all powers of the vertex cover ideal of $G$ have linear quotients.

Proof. Let $F_{1}, \ldots, F_{m}$ be the facets of $\Delta(G)$ with free vertices. We have $V(G)=F_{1} \cup \cdots \cup$ $F_{m}$ or $V(G) \backslash\{y\}=F_{1} \cup \cdots \cup F_{m}$ for some $y \in V(G)$. In order to prove that $J(G)^{k}$ has linear quotients, we show $J(G)^{k}$ is weakly polymatroidal with respect to the order

$$
y<y_{m}<\cdots<y_{1}<x_{n}<\cdots<x_{1}
$$

on the variables (corresponding to the vertices of $G$ ), where $y_{1}, \ldots, y_{m}$ are the free vertices of $G$ and $y, x_{1}, \ldots, x_{n}$ are non-free vertices of $G$.

First we note that given any minimal vertex cover $C$ of $G$ and for any facet $F_{i} \in$ $\mathcal{F}(\Delta(G))$, there exists a unique vertex $u_{i} \in F_{i}$ such that $u_{i} \notin C$ and $F_{i} \backslash\left\{u_{i}\right\} \subseteq C$.

Now let $f=f_{1} \cdots f_{k}$ and $g=g_{1} \cdots g_{k}$ be two elements in the minimal generating set of $J(G)^{k}$ such that $\operatorname{deg}_{z^{\prime}} f=\operatorname{deg}_{z^{\prime}} g$ for any variable $z^{\prime}>z$ and $\operatorname{deg}_{z} f>\operatorname{deg}_{z} g$. Thus there exists $t$ such that $z \notin \operatorname{supp}\left(g_{t}\right)$. Note that $z \neq y$, otherwise $\operatorname{deg}_{z^{\prime}} f=\operatorname{deg}_{z^{\prime}} g$ for any variable $z^{\prime} \neq y$ and $\operatorname{deg}_{y} f>\operatorname{deg}_{y} g$ which implies that $g$ divides $f$, which is a contradiction by our assumption that $f$ and $g$ belong to the minimal monomial set of generators of $J(G)^{k}$. Then in order to check the condition of weakly polymatroidals we consider the following cases:

Case 1. $z=x_{j}$ for some $j$ : Let $F_{i}$ be the facet of $\Delta(G)$ containing $x_{j}$. Since $\operatorname{supp}\left(g_{t}\right)$ is a minimal vertex cover of $G$, we have $F_{i} \backslash\left\{x_{i}\right\} \subseteq \operatorname{supp}\left(g_{t}\right)$. Therefore by substituting any free vertex $y_{\ell}$ of $F_{i} \cap \operatorname{supp}\left(g_{t}\right)$ with $x_{j}$ we get again a vertex cover of $G$ (not necessarily minimal). Hence

$$
g^{\prime}=g_{1} \cdots g_{t-1}\left(x_{j} g_{t} / y_{\ell}\right) g_{t+1} \cdots g_{k} \in J(G)^{k} .
$$

Case 2. $z=y_{j}$ for some $j$ : Let $F_{i}$ be the facet of $\Delta(G)$ containing $y_{j}$. First note that our assumption on $z$ (that $\operatorname{deg}_{x_{i}} f=\operatorname{deg}_{x_{i}} g$ and $\operatorname{deg}_{y_{s}} f=\operatorname{deg}_{y_{s}} g$ for all $i$ and all $s<j$ ), implies that the sets

$$
\left\{f_{m}: x_{i} \notin \operatorname{supp}\left(f_{m}\right) \text { for some } i, \text { or } y_{s} \notin \operatorname{supp}\left(f_{m}\right) \text { for some } s<j\right\}
$$


and

$$
\left\{g_{m}: x_{i} \notin \operatorname{supp}\left(g_{m}\right) \text { for some } i, \text { or } y_{s} \notin \operatorname{supp}\left(g_{m}\right) \text { for some } s<j\right\}
$$

have the same cardinality. This implies that there exists a free vertex $y_{\ell} \in F_{i}$ with $\ell>j$ since $\operatorname{deg}_{y_{j}} f>\operatorname{deg}_{y_{j}} g$. Therefore the subset $\left(\operatorname{supp}\left(g_{t}\right) \backslash\left\{y_{\ell}\right\}\right) \cup\left\{y_{j}\right\}$ is again a vertex cover of $G$ and so

$$
g^{\prime}=g_{1} \cdots g_{t-1}\left(y_{j} g_{t} / y_{\ell}\right) g_{t+1} \cdots g_{k}
$$

belongs to $J(G)^{k}$, as desired.

In [11] it is shown that a chordal graph $G$ is Cohen-Macaulay if and only if $V(G)$ is the disjoint union of the facets of $\Delta(G)$ with free vertices. Hence $\bigcup_{F \in \mathcal{F}(\Delta(G))} F=V(G)$. The following result is an extension of [10, Theorem 2.7]. Applying [15, Corollary 1.4] we have

Corollary 1.4. All powers of the vertex cover ideal of a graph $G$ with the property that $|V(G)|-1 \leq\left|\bigcup_{F \in \mathcal{F}(\Delta(G))} F\right|$ have componentwise linear quotients. In particular, they are componentwise linear.

Recall that the complete graph $K_{n}$ on $\left\{x_{1}, \ldots, x_{n}\right\}$ is a finite graph with $\left\{x_{i}, x_{j}\right\} \in E(G)$ for all $1 \leq i<j \leq n$. Let $G$ be a graph on the vertex set $\left\{x_{1}, \ldots, x_{n}, y_{1}, \ldots, y_{m}\right\}$ such that $\left\{x_{i}, x_{j}\right\} \in E(G)$ for all $i<j$ and $\left\{y_{i}, y_{j}\right\} \notin E(G)$ for all $i<j$. Then $G$ is called a star graph based on $K_{n}$. As a consequence of Theorem 1.3 we get [10, Theorem 2.3].

Corollary 1.5. All powers of the vertex cover ideal of a star graph based on $K_{n}$ are componentwise linear.

Proof. We have $V(G)=F_{1} \cup \cdots \cup F_{m} \cup F_{m+1}$, where $F_{\ell}=\left\{y_{\ell}\right\} \cup N\left(y_{\ell}\right)$ for $\ell=1, \ldots, m$ and $F_{m+1}=\left\{x_{i}: N\left(x_{i}\right) \subseteq\left\{x_{1}, \ldots, x_{n}\right\}\right\}$. Note that $y_{\ell}$ is a free vertex in the facet $F_{\ell}$ for $\ell=1, \ldots, m$, and in the case that $F_{m+1}$ is nonempty, each vertex in $F_{m+1}$ is a free vertex. Therefore the condition of Theorem 2.3 holds which completes the proof.

Example 1.6. Let $G$ be the graph depicted in the following picture. The free vertices have been marked by smaller nodes, and the vertex $v$ is the only vertex which does not belong to any facet $F$ with a free vertex. Thus by Theorem 1.3 all powers of the vertex cover ideals of $G$ are componentwise linear.

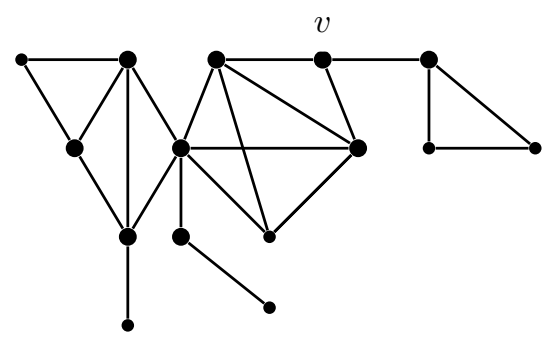

Example 1.7. Let $G$ be the chordal graph drawn below. The graph $G$ is neither a star graph nor a Cohen-Macaulay graph. But we have $\left|\bigcup_{F \in \mathcal{F}(\Delta(G))} F\right|=|V(G)|-1$. We consider the labeling described in Theorem 1.3 . Therefore all powers of the vertex cover ideals of $G$ are componentwise linear.

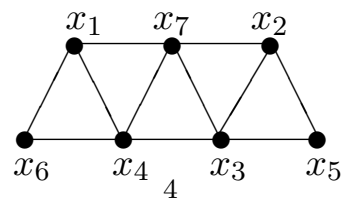




\section{Cohen-Macaulay cactus graphs}

In this section we give a combinatorial characterization for Cohen-Macaulay edge ideals among cactus graphs. A connected graph is a cactus graph if each edge belongs to at most one cycle. Note that the facets of $\Delta(G)$ in a cactus graph are 3-cycles and some edges. Sequentially Cohen-Macaulay cactus graphs are classified in [16, Theorem 2.8].

Theorem 2.1. A cactus graph $G$ is sequentially Cohen-Macaulay if and only if for each cycle $C_{m}, m \neq 3,5$, one of the following holds:

(i) $C_{m}$ has a common vertex $u$ with some clique $F$ of $G$, where $F$ has a free vertex $u^{\prime} \neq u$;

(ii) $C_{m}$ has a common vertex $u$ with some cycle $C_{5}$ such that for $u \in V\left(C_{5}\right) \cap V\left(C_{m}\right)$ and $v, w \in N_{C_{5}}(u)$ we have $\operatorname{deg}_{G}(v)=\operatorname{deg}_{G}(w)=2$.

A sequentially Cohen-Macaulay graph is Cohen-Macaulay if and only if it is unmixed, (see [19, 22]). Thus in order to classify Cohen-Macaulay cactus graphs we need to determine unmixed graphs among those sequentially Cohen-Macaulay graphs. First we prove the following technical lemma on sequentially Cohen-Macaulay cactus graphs. A vertex $v$ of $G$ is called an adjacent vertex to a cycle $C_{m}$ if $v$ is adjacent to a vertex $u \in V\left(C_{m}\right)$.

Lemma 2.2. Let $G$ be a sequentially Cohen-Macaulay cactus graph. Then one of the following statements holds:

(a) $G$ has a free vertex;

(b) There is a cycle $C_{5}$ with no adjacent vertex of degree greater than two.

Proof. The proof is by induction on the number of the vertices of $G$. If $|V(G)| \leq 5$, then $G$ has a free vertex or $G$ is indeed a 5 -cycle. By contrary assume that $G$ is a sequentially Cohen-Macaulay cactus graph with the smallest number of vertices which does not fulfill the conditions of theorem. Since $G$ does not have a free vertex, by Dirac's Theorem $G$ is not a chordal graph and so it has a chordless cycle of length greater than three. If there exists a cycle $C_{m}$ with $m \neq 5$, then by Theorem 2.1(ii) there exists a cycle $C_{5}$ on the vertices $x_{1}, \ldots, x_{5}$ adjacent to $C_{m}$ with $x_{1} \in V\left(C_{5}\right) \cap V\left(C_{m}\right)$ and $\operatorname{deg}_{G}\left(x_{2}\right)=\operatorname{deg}_{G}\left(x_{5}\right)=2$. By our (contrary) assumption we have $\operatorname{deg}_{G}\left(x_{3}\right)>2$ and $\operatorname{deg}_{G}\left(x_{4}\right)>2$.

Let $G^{\prime}$ be the contraction of the vertices of $C_{m}$ in $G$. Since $G$ is a cactus graph, $G^{\prime}$ is again a cactus graph with fewer vertices. Next we show that $G^{\prime}$ is sequentially CohenMacaulay. Any cycle $C_{m}$ in $G^{\prime}$ with $m \neq 3,5$, is indeed a cycle in $G$. Therefore there exists a cycle $C_{5}$ adjacent to $C_{m}$ with desired property as Theorem 2.1(ii), since $G$ has no free vertex. Note that the contraction of $C_{m}$ does not remove any vertex of $C_{5}$ and this process does not decrease the degree of any vertex in $V(G) \backslash V\left(C_{m}\right)$. Hence $G^{\prime}$ has no free vertex. Moreover, contraction does not change the degrees of the vertices of degree two in the induced subgraph on the vertices $V(G) \backslash V\left(C_{m}\right)$. Therefore the condition (ii) of Theorem 2.1 holds for each cycle $C_{m}$ in $G^{\prime}$. So $G^{\prime}$ has a 5 -cycle which is also in $G$, with the property desired in (b), a contradiction.

In the case that there exists no $C_{m}$ with $m \neq 5$, we have a cycle $C_{5}$ in $G$, since $G$ is not chordal. We consider $G^{\prime}$ as the contraction of the vertices of $C_{5}$ in $G$. Then the same argument completes the proof.

Theorem 2.3. For a cactus graph $G$, let $F_{1}, \ldots, F_{m}$ be the facets of $\Delta(G)$ with some free vertices and $G_{1}, \ldots, G_{n}$ be the 5 -cycles of the induced subgraph on the vertices $V(G) \backslash$ $V\left(F_{1} \cup \cdots \cup F_{m}\right)$ with no adjacent vertex of degree greater than two in $G$, and $L_{1}, \ldots, L_{t}$ 
be the edges in the induced subgraph on the vertices

$$
V(G) \backslash V\left(F_{1} \cup \cdots \cup F_{m} \cup G_{1} \cup \cdots \cup G_{n}\right)
$$

such that each $L_{i}$ belongs to some 4 -cycle in $G$. Then the following statements are equivalent:

(a) $G$ is Cohen-Macaulay;

(b) $V(G)$ is the disjoint union of the vertices of $F_{1}, \ldots, F_{m}, G_{1}, \ldots, G_{n}, L_{1}, \ldots, L_{t}$.

In order to formulate the main result of this section we first fix our notation.

Notation. With the hypothesis of Theorem 2.3 (with no loss of generality) we assume that $F_{i}=\left\{x_{i 1}, \ldots, x_{i k_{i}}\right\}$, where $x_{i b_{i}}, \ldots, x_{i k_{i}}=u_{i}$ are the free vertices of $F_{i}$ for all $i$. Consider a labeling on the vertices of the 5 -cycle $G_{j}$ as $y_{j 1}, y_{j 2}, \ldots, y_{j 5}$ such that the degrees of $y_{j 3}, y_{j 4}, y_{j 5}$ are two for all $j$. Assume that $z_{i 1}, z_{i 2}$ are the vertices of $L_{i}$ for all $i$.

Example 2.4. Let $G$ be the cactus graph depicted in the following figure. The labeling given on the vertices of $G$ is of the form which we described above. Note that each minimal vertex cover $C$ of $G$ is of the form

$$
C=\left\{x_{1 i}, x_{1 j}, x_{2 k}, y_{r}, y_{s}, y_{t}, z_{m}\right\}
$$

for some $1 \leq i \leq j \leq 3,1 \leq k \leq 2,1 \leq r \leq s \leq t \leq 5$ and $m=1$ or 2 . Note that according to Theorem 2.3 this graph is Cohen-Macaualy.

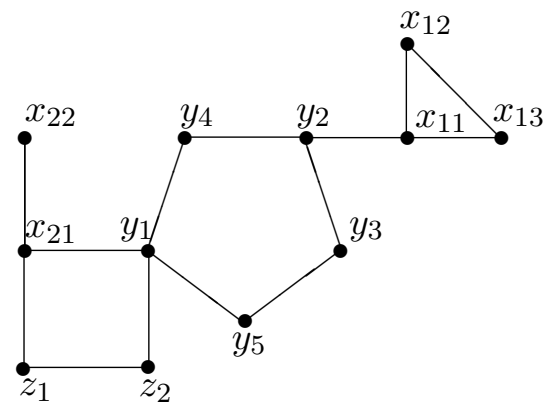

Before giving the proof of the theorem we need the following technical lemma (in which we use the similar notation as Theorem 2.3.

Lemma 2.5. Let $G$ be a Cohen-Macaulay cactus graph and

$$
B=V(G) \backslash V\left(F_{1} \cup \cdots \cup F_{m} \cup G_{1} \cup \cdots \cup G_{n} \cup L_{1} \cup \cdots \cup L_{t}\right) .
$$

Then $\left.G\right|_{B}$, the induced subgraph $G$ on $B$ is a Cohen-Macaulay cactus graph.

Proof. The induced subgraph $\left.G\right|_{B}$ is again a cactus graph. First we show that $\left.G\right|_{B}$ is sequentially Cohen-Macaulay. By Theorem 2.1 it is enough to show that each cycle $C_{m}$ in $\left.G\right|_{B}$ with $m \neq 3,5$ has some adjacent free vertex or some adjacent 5 -cycle fulfilling the condition (ii) of Theorem 2.1. Each cycle $C_{m}$ in $\left.G\right|_{B}$ with $m \neq 3,5$ is indeed a cycle in $G$. Therefore it has an adjacent free vertex $v$ or an adjacent 5-cycle $C$ as (ii). If $v \in F_{i}$ for some $i$, then the vertex of $C_{m}$ adjacent to $v$ is not in $G^{\prime}$ and so $C_{m}$ is not a cycle in $G^{\prime}$, a contradiction. Let $V(C)=\left\{x_{1}, \ldots, x_{5}\right\}, x_{1} \in V(C) \cap V\left(C_{m}\right)$ and $\operatorname{deg}\left(x_{2}\right)=\operatorname{deg}\left(x_{5}\right)=2$. Since $C_{m}$ is a cycle in $\left.G\right|_{B}$ and $x_{1} \in V\left(\left.G\right|_{B}\right)$, we have $C \neq G_{j}$ for all $j$. Moreover the 
vertices $x_{2}$ and $x_{5}$ are in $V\left(\left.G\right|_{B}\right)$, since $\operatorname{deg}\left(x_{2}\right)=\operatorname{deg}\left(x_{5}\right)=2$. If $x_{3}$ is not in $V\left(\left.G\right|_{B}\right)$, then $x_{2}$ is a vertex of degree one adjacent to $C_{m}$. If $x_{4}$ is not in $V\left(\left.G\right|_{B}\right)$, then $x_{5}$ is a vertex of degree one adjacent to $C_{m}$, as desired. Therefore one of the statements (i) or (ii) of Theorem 2.1 holds for $C_{m}$ and so $\left.G\right|_{B}$ is sequentially Cohen-Macaulay.

Now we show that $\left.G\right|_{B}$ is unmixed. The set

$$
M_{1}=\left\{y_{11}, y_{12}, y_{13}, \ldots, y_{n 1}, y_{n 2}, y_{n 3}, z_{11}, \ldots, z_{t 1}\right\} \cup V\left(F_{1} \backslash\left\{u_{1}\right\} \cup \cdots \cup F_{m} \backslash\left\{u_{m}\right\}\right)
$$

is a minimal vertex cover of the induced subgraph on $V(G) \backslash B$. For any minimal vertex cover $X$ of $\left.G\right|_{B}$,

$$
M=X \cup M_{1}
$$

is a vertex cover of $G$, since $\left\{u_{i}, b\right\},\left\{y_{j 4}, b\right\}$ and $\left\{y_{j 5}, b\right\}$ are not in $E(G)$ for all $i, j$, and all $b \in B$. Moreover, all neighbors of $z_{i 2}$ are already in $M_{1}$. Let $M^{\prime} \subseteq M$ be a minimal vertex cover of $G$. Assume that the 4 -cycle consisting $L_{i}$ has the vertices $z_{i 1}, z_{i 2}, z_{i 3}, z_{i 4}$, where $z_{i 3}, z_{i 4} \in V\left(F_{1} \cup \cdots \cup F_{m} \cup G_{1} \cup \cdots \cup G_{n}\right)$ for each $i$. If $z_{i 3} \in V\left(F_{\ell}\right)$, then $z_{i 3} \in M^{\prime}$, since $u_{\ell}$ and $z_{i 3}$ are adjacent. If $z_{i \ell} \in V\left(G_{\ell}\right)$, then $z_{i \ell} \in M^{\prime}$, since one of the vertices $y_{\ell 4}, y_{\ell 5}$ which are not in $M$ are adjacent to $z_{i 3}$. Hence $z_{i 3} \in M^{\prime}$. Similarly $z_{i 4} \in M$. Since $\left\{z_{i 1}, z_{i 2}\right\} \in E(G)$ and $z_{i 1} \notin M_{1}$ we have $z_{i 2} \in X$. Hence $M^{\prime}=M$ which implies that $\left.G\right|_{B}$ is unmixed.

Now we are ready to state the proof of the theorem.

Proof of Theorem 2.3. (a) $\Rightarrow$ (b): The proof is by induction on the number of the vertices of $G$. Let

$$
B=V(G) \backslash V\left(F_{1} \cup \cdots \cup F_{m} \cup G_{1} \cup \cdots \cup G_{n} \cup L_{1} \cup \cdots \cup L_{t}\right) .
$$

By contrary assume that $B \neq \emptyset$. The induced subgraph $\left.G\right|_{B}$ is again a Cohen-Macaulay cactus graph by Lemma 2.5 . So by induction hypothesis

$$
B=V\left(H_{1} \cup \cdots \cup H_{s} \cup K_{1} \cup \cdots \cup K_{r} \cup P_{1} \cup \cdots \cup P_{h}\right)
$$

where $H_{1}, \ldots, H_{s}$ are the facets of $\Delta\left(\left.G\right|_{B}\right)$ with some free vertices, and $K_{1}, \ldots, K_{r}$ are the 5 -cycles of the induced subgraph on the vertices $B \backslash V\left(H_{1} \cup \cdots \cup H_{s}\right)$ with no adjacent vertex of degree greater than two in $G$, and $P_{1}, \ldots, P_{h}$ are the edges belonging to some 4-cycle in $\left.G\right|_{B}$. Let $v_{i}$ be a free vertex of $H_{i}$ in $\left.G\right|_{B}$. For each 5 -cycle $K_{j}$ with vertices $y_{j 1}, y_{j 2}, \ldots, y_{j 5}$ assume that the degrees of $y_{j 3}, y_{j 4}, y_{j 5}$ are two in $\left.G\right|_{B}$ and $z_{i 1}, z_{i 2}$ are the vertices of $P_{i}$ for all $i$. Then

$$
M_{2}=\left\{y_{1,1}, y_{1,2}, y_{1,3}, \ldots, y_{r, 1}, y_{r, 2}, y_{r, 3}, z_{1,1}, \ldots, z_{h, 1}\right\} \cup V\left(H_{1} \backslash\left\{v_{1}\right\} \cup \cdots \cup H_{s} \backslash\left\{v_{s}\right\}\right)
$$

is a minimal vertex cover of $\left.G\right|_{B}$ of size $|B|-s-2 r-h$. Since

$$
Z=M_{1} \cup M_{2}
$$

is a minimal vertex cover of $G$ and $G$ is unmixed, each minimal vertex cover of $G$ consists of $n-(m+s+2 k+2 r+t+h)$ vertices.

Let $Y$ be a minimal vertex cover of $G$. If $F_{i} \subset Y$, then $Y \backslash\left\{u_{i}\right\}$ is a vertex cover of $G$ for each free vertex $u_{i} \in F_{i}$. Therefore $\left|Y \cap V\left(F_{i}\right)\right|=\left|F_{i}\right|-1$ for all minimal vertex covers $Y$ of $G$ and for all $i$. Since $Y$ is a minimal vertex cover of $G,\left|Y \cap V\left(G_{j}\right)\right|=3$. Otherwise there exists a vertex of degree two in $G_{j}$ such that its neighbors are all in $Y$, a contradiction (by the minimality assumption on $Y$ ). Assume that the 4-cycle corresponding to $L_{i}$ has the vertices $z_{i 1}, \ldots, z_{i 4}$, where $z_{i 1}, z_{i 2} \in L_{i}$. Since at least one of the vertices $z_{i 3}, z_{i 4}$ is in $Y$, we have $\left|Y \cap V\left(L_{i}\right)\right|=1$. 
Moreover, $\left|Y \cap V\left(H_{i}\right)\right| \geq\left|H_{i}\right|-1,\left|Y \cap V\left(K_{j}\right)\right| \geq 3$ and $\left|Y \cap V\left(P_{\ell}\right)\right| \geq 1$ for all $i, j, \ell$. Since $|Y \cap B|=|B|-s-2 r-h$, we have $\left|Y \cap V\left(H_{i}\right)\right|=\left|H_{i}\right|-1,\left|Y \cap V\left(K_{j}\right)\right|=3$ and $\left|Y \cap V\left(P_{\ell}\right)\right|=1$ for all $i, j, \ell$.

Now we show that $V\left(F_{i}\right) \cap V\left(F_{j}\right)=\emptyset$ for all $i, j$. By contradiction assume that $w \in$ $V\left(F_{i_{1}}\right) \cap \cdots \cap V\left(F_{i_{\ell}}\right)$. Let $v_{i_{j}} \in V\left(F_{i_{j}}\right) \backslash Y$ be a free vertex for $j=1, \ldots, \ell$. Then $\left(Y \cup\left\{v_{i_{1}}, \ldots, v_{i_{\ell}}\right\}\right) \backslash\{w\}$ is a minimal vertex cover of $G$ consisting of $|Y|+\ell-1$ vertices, a contradiction.

Now, we claim that $r=0$. By contrary assume that $K_{1}$ is a 5 -cycle with the vertices $y_{1}, \ldots, y_{5}$ belonging to $B \backslash V\left(H_{1} \cup \cdots \cup H_{s}\right)$. Therefore, $K_{1}$ has a pair of adjacent vertices of degree greater than two in $G$ such that at least one of them has some neighbor in $V(G) \backslash B$ or in $V\left(H_{1} \cup \cdots \cup H_{s}\right)$. Assume that $y_{2}, y_{3}$ are some vertices of degree greater than two in $G$. Consider $a \in N_{G}\left(y_{2}\right)$ and $b \in N_{G}\left(y_{3}\right)$, where $a \in(V(G) \backslash B) \cup V\left(H_{1} \cup \cdots \cup H_{s}\right)$ and $b \neq y_{2}, y_{5}$. Since $G$ is a cactus graph, $a \neq b$ and they are not adjacent. Also $\left\{y_{1}, a\right\},\left\{y_{1}, b\right\}$ are not in $E(G)$. Therefore, there exists a minimal vertex cover $X$ of $G$ with $X \subset V(G) \backslash\left\{a, b, y_{1}\right\}$. Therefore $\left\{y_{2}, y_{3}, y_{4}, y_{5}\right\} \subset X$ which is a contradiction by our observation that $\left|K_{1} \cap X\right|=3$. Hence we have $r=0$.

By induction hypothesis we have $V\left(H_{i}\right) \cap V\left(H_{j}\right)=\emptyset$ for all $i, j$. Since $H_{1}$ is a non-facet of $\Delta(G)$ or a facet with no free vertex, (for each free vertex $\delta$ of $H_{1}$ in $\Delta\left(\left.G\right|_{B}\right.$ ), there is $a \in V(G) \backslash B$ with $\{\delta, a\} \in E(G))$. Let $\delta_{1}, \ldots, \delta_{\ell}$ be the free vertices of $H_{1}$ and $v_{1}, \ldots, v_{d}$ be the non-free vertices of $H_{1}$. Set $A=\left\{a_{1}, \ldots, a_{\ell}, b_{1}, \ldots, b_{d}\right\}$, where $a_{l} \in V(G) \backslash B$ is an adjacent vertex to $\delta_{\ell}$ (for each $\ell$ ) and $b_{i} \in B \backslash H_{1}$ is an adjacent vertex to $v_{i}$. Since $G$ is a cactus graph, each pair of the vertices in $A$ are non-adjacent. So there exists a minimal vertex cover $X$ of $G$ with $X \subseteq V(G) \backslash A$. In this case $H_{1} \subseteq X$ which is a contradiction by the fact that $\left|X \cap H_{1}\right|=\left|H_{1}\right|-1$. Therefore $s=0$ which implies that $B=\emptyset$, since two vertices of $P_{1}$ should be in $V\left(H_{1} \cup \cdots \cup H_{s}\right) \cup V\left(K_{1} \cup \cdots \cup K_{r}\right)$ which is empty.

Next we show that for all $i, j$, we have

$$
V\left(G_{i}\right) \cap V\left(G_{j}\right)=\emptyset .
$$

By contrary assume that $V\left(G_{i}\right) \cap V\left(G_{j}\right) \neq \emptyset$ for some $i, j$. Since $G$ is a cactus graph, we have $\left|V\left(G_{i}\right) \cap V\left(G_{j}\right)\right|=1$, say $V\left(G_{i}\right) \cap V\left(G_{j}\right)=\{y\}$, as depicted in the following picture.

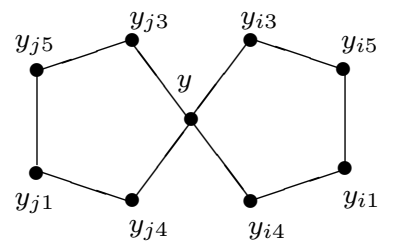

Let $A=\left\{i: y \in V\left(G_{i}\right)\right\}$ and $V\left(G_{i}\right)=\left\{y, y_{i 2}, y_{i 3}, y_{i 4}, y_{i 5}\right\}$ for each $i \in A$. Since $\operatorname{deg}(y)>2$, we can assume that $\operatorname{deg}\left(y_{i 3}\right)=\operatorname{deg}\left(y_{i 4}\right)=\operatorname{deg}\left(y_{i 5}\right)=2$ for all $i \in A$.

Since $V(G)=V\left(F_{1} \cup \cdots \cup F_{m} \cup G_{1} \cup \cdots \cup G_{n} \cup L_{1} \cup \cdots \cup L_{t}\right)$, for each vertex $v \in$ $N_{G}(y) \backslash\left(\cup_{j \in A} G_{j}\right)$ there exists some $i$ such that $v$ belongs to $F_{i}$ or the 4-cycle corresponding to $L_{i}$. Let

$$
B=\left\{v: v \in N_{G}(y) \cap V\left(F_{1} \cup \cdots \cup F_{m} \cup L_{1} \cup \cdots \cup L_{t}\right)\right\} .
$$

Assume that $F_{i_{1}}, \ldots, F_{i_{\ell}}$ are all facets with some common vertex with $N_{G}(y)$. Let $v_{i_{j}}$ be a free vertex of $F_{i_{j}}$ for $j=1, \ldots, \ell$. Assume that $y$ belongs to the 4 -cycles $L_{j_{1}}^{\prime}, \ldots, L_{j_{s}}^{\prime}$, 
where $L_{j_{\ell}}^{\prime}$ is the 4-cycle containing the edge $L_{j_{\ell}}$. Then $B$ contains some non-free vertices of $F_{i_{1}}, \ldots, F_{i_{\ell}}$ and also the neighbors of $y$ in $L_{j_{1}}^{\prime}, \ldots, L_{j_{s}}^{\prime}$. Consider the subset

$$
C=\left\{v_{i_{1}}, \ldots, v_{i_{\ell}}, z_{j_{1}}, \ldots, z_{j_{s}}\right\},
$$

where for all $\ell, z_{j \ell}$ is a vertex of $L_{j \ell}^{\prime}$ which is not adjacent to $y$.

Since $G$ is a cactus graph, no vertex of $C$ is adjacent to $G_{j}$ for $j \in A$. Note that each vertex $z_{j \ell}$ is of degree two and so it is not adjacent to any vertex of $C$. Also $v_{i_{j}}$ is not adjacent to any vertex of $C$, since $v_{i_{j}}$ is a free vertex. Therefore, each pair of the vertices in $C$ are not adjacent and so there exists a minimal vertex cover $X$ of the induced subgraph on the vertices $V(G) \backslash\left(\cup_{i \in A} V\left(G_{i}\right)\right)$ such that

$$
X \subset V(G) \backslash\left(C \cup\left(\cup_{i \in A} V\left(G_{i}\right)\right)\right) .
$$

Then all non-free vertices of $F_{i_{1}}, \ldots, F_{i_{\ell}}$ and the neighbors of $y$ in $L_{j_{1}}^{\prime}, \ldots, L_{j_{s}}^{\prime}$ should be in $X$ which implies that $B \subset X$. Let

$$
Y_{1}=X \cup\{y\} \cup\left(\cup_{i \in A}\left\{y_{i 1}, y_{i 3}\right\}\right) \quad \text { and } \quad Y_{2}=X \cup\left(\cup_{i \in A}\left\{y_{i 1}, y_{i 3}, y_{i 4}\right\}\right) .
$$

Since $y_{i 4}$ and $y_{i 5}$ are non-adjacent vertices of degree two, and $z_{j_{\ell}}$ is just adjacent to some neighbors of $u$ belonging to $X, Y_{1}$ is a minimal vertex cover of $G$. We have $N_{G}(u)=$ $B \cup\left(\cup_{i \in A}\left\{y_{i 3}, y_{i 4}\right\}\right)$ which implies that $Y_{2}$ is a minimal vertex cover of $G$. But $Y_{1}$ and $Y_{2}$ have the same size if and only if $k=1$, a contradiction.

(b) $\Rightarrow$ (a): Let that $X \subset V(G)$ be a minimal vertex cover of $G$. Then we have $\mid X \cap$ $V\left(F_{i}\right)|=| F_{i} \mid-1$ and $\left|X \cap V\left(G_{j}\right)\right|=3$ for all $i, j$. Let $L_{i}^{\prime}$ be the 4-cycle on the vertices $z_{i 1}, z_{i 2}, z_{i 3}, z_{i 4}$, where $z_{i 1}$ and $z_{i 2}$ are of degree two. Since $\left\{z_{i 3}, z_{i 4}\right\} \in E(G)$, at least one of these vertices belongs to $X$. Assume that $z_{i 3} \in X$. Since $N\left(z_{i 2}\right)=\left\{z_{i 1}, z_{i 3}\right\}$ and $X$ is a minimal vertex cover, we can not have both $z_{i 1}, z_{i 2}$ in $X$ which implies that $\left|X \cap L_{i}\right|=1$. Therefore $|X|=n-2 k-m-t$ for each vertex cover $X$ of $G$ and so $G$ is unmixed. Let $C_{m}$ be a cycle in $G$ with $m \neq 3,5$. Then $C_{m}$ has a common vertex $v$ with some $F_{i}$ or $G_{j}$, since $V(G)$ is the disjoint union of the vertices of $F_{1}, \ldots, F_{m}, G_{1}, \ldots, G_{n}, L_{1}, \ldots, L_{t}$. If $v \in V\left(F_{i}\right)$ for some $i$, then (i) holds in Theorem 2.1. If $v \in V\left(G_{j}\right)$, then the vertices of $G_{j}$ adjacent to $v$ are of degree two, since $G_{j}$ has no adjacent vertex of degree greater than two in $G$ which implies (ii) in Theorem 2.1.

Example 2.6. Let $G$ be the graph depicted in the following picture. Then $G \backslash\{v\}$ and $G \backslash(\{v\} \cup N(v))$ are both cactus graphs which are Cohen-Macaulay by Theorem 2.3. Thus $G$ is sequentially Cohen-Macaulay (see, e.g., [21, Lemma 2.4]). One can easily check that $G$ is also unmixed and hence Cohen-Macaulay.

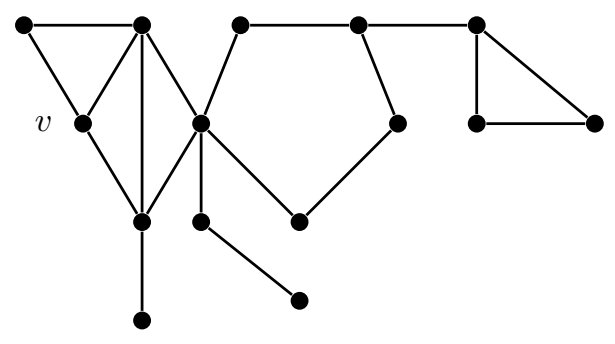




\section{Vertex COVER ideal of a COHEn-Macaulay CaCtus Graph}

The vertex cover ideals of Cohen-Macaulay graphs are studied for bipartite graphs and chordal graphs, see [8, 10, 5, 14. In all cases there are some combinatorial properties of $G$ which guarantee being Cohen-Macaulay. Moreover all powers of the vertex cover ideal of $G$ are weakly polymatroidal with respect to the natural lexicographical ordering on the variables associated to the vertices of $G$. More precisely, $J(G)^{k}$ is weakly polymatroidal for each $k$ when $G$ is of one of the following types:

(1) Cohen-Macaulay bipartite graph (see [8] and [15, Theorem 2.2]);

(2) Cohen-Macaulay chordal graph (see [10, Theorem 2.7] and [14, Theorem 1.7]).

As a consequence of Eagon and Reiner's Theorem from [2], we have that a graph is Cohen-Macaulay if and only if its vertex cover ideal has a linear resolution. By the above consideration we expect that all powers of the vertex cover ideal of a Cohen-Macaulay graph have the same property, i.e. they have linear resolutions. We will choose an order on the vertices of a Cohen-Macaulay graph such that under this ordering $J(G)^{k}$ admits weakly polymatroidal condition for each $k$. Thus we pose the following question.

Question 3.1. Do all powers of the vertex cover ideal of a Cohen-Macaulay graph have linear resolutions?

In this section we study the above question for cactus graphs, and we show that in the case of cactus graphs the answer is yes. Indeed using the combinatorial characterization of Cohen-Macaulay cactus graph given in the previous section we show that all powers of the vertex cover ideals of these graphs have linear quotients.

Example 3.2. Returning to Example 2.4, before stating the main theorem of this section, we overview why the ideal $J(G)^{2}$ is weakly polymatroidal. First we consider the lexicographical ordering induced by the total ordering

$$
x_{11}>x_{12}>x_{13}>x_{21}>x_{22}>y_{1}>y_{2}>y_{3}>y_{4}>y_{5}>z_{1}>z_{2}
$$

on the variables (corresponding to the vertices of $G$ ). Consider the monomials

$$
f=x_{11}^{2} x_{12}^{2} x_{21}^{2} y_{1}^{2} y_{2}^{2} y_{3}^{2} z_{1}^{2} \quad \text { and } \quad g=x_{11}^{2} x_{12}^{2} x_{21}^{2} y_{1} y_{2} y_{3}^{2} y_{4} y_{5} z_{1} z_{2}
$$

of $J(G)^{2}$ with $f>_{l e x} g$. As we see, the variable $y_{1}$ is the greatest variable which has the higher exponent in $f$ than $g$. Then we decompose $g$ as $g=g_{1} g_{2}$ in which $g_{1}=$ $x_{11} x_{12} x_{21} y_{3} y_{4} y_{5} z_{2}$ and $g_{2}=x_{11} x_{12} x_{21} y_{1} y_{2} y_{3} z_{1}$. Then the fact that $\operatorname{supp}\left(g_{2}\right)$ is a minimal vertex cover of $G$ together with $y_{1} \notin \operatorname{supp}\left(g_{2}\right)$ implies that $N_{G}\left(y_{1}\right)=\left\{y_{4}, y_{5}\right\}$ is a subset of $\operatorname{supp}\left(g_{2}\right)$. Since $y_{2}$ and $y_{2}$ are adjacent we have $\left|\left\{y_{2}, y_{3}\right\} \cap C\right| \geq 1$. On the other hand $\left\{y_{4}, y_{5}\right\} \subseteq \operatorname{supp}\left(g_{2}\right)$ implies that only one of the vertices $y_{2}$ or $y_{3}$ belongs to $C$ (since $C$ is a minimal vertex cover of $G)$. As we see $y_{3} \in C$. Then $g_{2}^{\prime}=y_{1} g_{2} / y_{5}$ is again in $J(G)$. Thus $g^{\prime}=g_{1} g_{2}^{\prime}$ belongs to $J(G)^{2}$ which fulfills the condition of weakly polymatroidal.

Theorem 3.3. All powers of the vertex cover ideal of a Cohen-Macaulay cactus graph are weakly polymatroidal. In particular they have linear resolutions.

Proof. Let $V(G)$ be the disjoint union of $F_{1}, \ldots, F_{m}, G_{1}, \ldots, G_{n}, L_{1}, \ldots, L_{t}$ (with the same notation of Theorem 2.3). Consider the lexicographical ordering induced by the following total ordering on the variables (corresponding to the vertices of $G$ ):

$$
\begin{gathered}
x_{11}>\cdots>x_{1 k_{1}}>\cdots>x_{m 1}>\cdots>x_{m k_{m}}>y_{11}>\cdots>y_{15}> \\
\cdots>y_{n 1}>\cdots>y_{n 5}>z_{11}>z_{12}>\cdots>z_{t 1}>z_{t 2}
\end{gathered}
$$


where $F_{i}=\left\{x_{i 1}, \ldots, x_{i k_{i}}\right\}$ and $x_{i b_{i}}, \ldots, x_{i k_{i}}$ are the free vertices of $F_{i}$ for all $i$, and $y_{j 1}, y_{j 4}, y_{j 2}, y_{j 3}, y_{j 5}$ are the vertices of $G_{j}$ such that the vertices $y_{j 3}, y_{j 4}, y_{j 5}$ are of degree two for all $j$, and $z_{i 1}, z_{i 2}$ are the vertices of $L_{i}$ of degree two for all $i$. First note that given any minimal vertex cover $C$ of $G$ and for any facet $F_{i} \in \mathcal{F}(\Delta(G)$ ), there exists a unique vertex $x_{i j} \in F_{i}$ such that $x_{i j} \notin C$ and $F_{i} \backslash\left\{x_{i j}\right\} \subseteq C$. More precisely, each minimal vertex cover of $G$ has exactly $k_{i}-1$ vertices of each $F_{i}$, three vertices of each $G_{j}$ and one vertex of each $L_{i}$.

Now, let $f=f_{1} \cdots f_{k}$ and $g=g_{1} \cdots g_{k}$ be two elements in the minimal generating set of $J(G)^{k}$ such that $\operatorname{deg}_{z^{\prime}} f=\operatorname{deg}_{z^{\prime}} g$ for any variable $z^{\prime}>z$ and $\operatorname{deg}_{z} f>\operatorname{deg}_{z} g$. Let $g_{j}$ be a monomial such that $z \notin \operatorname{supp}\left(g_{j}\right)$. Then we consider the following cases. In each case we will find $g_{j}^{\prime}>_{\text {lex }} g_{j}$ and

$$
g=g_{1} \cdots g_{j-1} g_{j}^{\prime} g_{j+1} \cdots g_{k}
$$

has desired properties (of weakly polymatroidal ideals) in $J(G)^{k}$. Hence $J(G)^{k}$ is a weakly polymatroidal ideal.

Case 1. $z=x_{i \ell}$ for some $i, \ell$ : In each minimal vertex cover $C$ of $G$ exactly one vertex of $F_{i}$ is missed. Therefore $\ell \neq k_{i}$ (otherwise we will have $\operatorname{deg}_{z}(f)=\operatorname{deg}_{z}(g)$ ). Since $\ell \neq k_{i}$ the subset $\left(\operatorname{supp}\left(g_{j}\right) \backslash\left\{x_{i k_{i}}\right\}\right) \cup\left\{x_{i \ell}\right\}$ is again a minimal vertex cover of $G$ and $g_{j}^{\prime}=x_{i \ell} g_{j} / x_{i k_{i}} \in J(G)$.

Case 2. $z=y_{i 1}$ for some $i$ : $\operatorname{Since} \operatorname{supp}\left(g_{j}\right)$ is a minimal vertex cover of $G$ and $y_{i 4}, y_{i 5} \in N_{G}\left(y_{i 1}\right)$, we have $y_{i 4}, y_{i 5} \in \operatorname{supp}\left(g_{j}\right)$. On the other hand $\left|V\left(G_{i}\right) \cap \operatorname{supp}\left(g_{j}\right)\right|=3$. Hence $y_{i 2}$ or $y_{i 3}$ belongs to $\operatorname{supp}\left(g_{j}\right)$. If $y_{i 2} \in \operatorname{supp}\left(g_{j}\right)$, then we set $g_{j}^{\prime}=g_{j} y_{i 1} / y_{i 4}$. Since $N_{G}\left(y_{i 4}\right)=\left\{y_{i 1}, y_{i 2}\right\} \subseteq \operatorname{supp}\left(g_{j}^{\prime}\right)$, we deduce that $\operatorname{supp}\left(g_{j}^{\prime}\right)$ is a minimal vertex cover of $G$. If $y_{i 3} \in \operatorname{supp}\left(g_{j}\right)$, then $g_{j}^{\prime}=g_{j} y_{i 1} / y_{i 5}$ has desired property, since $N_{G}\left(y_{i 5}\right)=\left\{y_{i 1}, y_{i 3}\right\} \subseteq$ $\operatorname{supp}\left(g_{j}^{\prime}\right)$ which implies that $\operatorname{supp}\left(g_{j}^{\prime}\right)$ is a minimal vertex cover of $G$.

Case 3. $z=y_{i 2}$ for some $i$ : Since $\operatorname{supp}\left(g_{j}\right)$ is a minimal vertex cover of $G$, we have $y_{i 3}, y_{i 4} \in \operatorname{supp}\left(g_{j}\right)$. Also $y_{i 1}$ or $y_{i 5}$ belongs to $\operatorname{supp}\left(g_{j}\right)$. If $y_{i 1} \in \operatorname{supp}\left(g_{j}\right)$, then we set $g_{j}^{\prime}=g_{j} y_{i 2} / y_{i 4}$. Then $\operatorname{supp}\left(g_{j}^{\prime}\right)$ is a minimal vertex cover of $G$, since $N_{G}\left(y_{i 4}\right)=\left\{y_{i 1}, y_{i 2}\right\} \subseteq$ $\operatorname{supp}\left(g_{j}^{\prime}\right)$. If $y_{i 5} \in \operatorname{supp}\left(g_{j}\right)$, then set $g_{j}^{\prime}=g_{j} y_{i 2} / y_{i 3}$. Since $N_{G}\left(y_{i 3}\right)=\left\{y_{i 2}, y_{i 5}\right\} \subseteq \operatorname{supp}\left(g_{j}^{\prime}\right)$, $\operatorname{supp}\left(g_{j}^{\prime}\right)$ is a minimal vertex cover of $G$.

Case 4. $z=y_{i 3}$ for some $i$ : Each minimal vertex cover $C$ contains one of the subsets

$$
\left\{y_{i 1}, y_{i 2}, y_{i 5}\right\},\left\{y_{i 2}, y_{i 4}, y_{i 5}\right\},\left\{y_{i 1}, y_{i 2}, y_{i 3}\right\},\left\{y_{i 1}, y_{i 3}, y_{i 4}\right\},\left\{y_{i 3}, y_{i 4}, y_{i 5}\right\} \text {. }
$$

Since $\operatorname{deg}_{y_{i 1}} f=\operatorname{deg}_{y_{i 1}} g$ and $\operatorname{deg}_{y_{i 2}} f=\operatorname{deg}_{y_{i 2}} g$ we have

$$
\left|\left\{f_{\ell}:\left\{y_{i 1}, y_{i 3}, y_{i 4}\right\} \subset \operatorname{supp}\left(f_{\ell}\right)\right\}\right|=\left|\left\{g_{\ell}:\left\{y_{i 1}, y_{i 3}, y_{i 4}\right\} \subset \operatorname{supp}\left(g_{\ell}\right)\right\}\right| \text {. }
$$

and also

$$
\left|\left\{f_{\ell}:\left\{y_{i 2}, y_{i 4}, y_{i 5}\right\} \subset \operatorname{supp}\left(f_{\ell}\right)\right\}\right|=\left|\left\{g_{\ell}:\left\{y_{i 2}, y_{i 4}, y_{i 5}\right\} \subset \operatorname{supp}\left(g_{\ell}\right)\right\}\right| .
$$

So there exists some $g_{j}$ with $\left\{y_{i 1}, y_{i 2}, y_{i 5}\right\} \subseteq \operatorname{supp}\left(g_{j}\right)$. Now we set $g_{j}^{\prime}=g_{j} y_{i 3} / y_{i 5}$.

Case 5. $z=y_{i 4}$ or $y_{i 5}$ for some $i$ : Since $\operatorname{deg}_{y_{i 1}} f=\operatorname{deg}_{y_{i 1}} g, \operatorname{deg}_{y_{i 2}} f=\operatorname{deg}_{y_{i 2}} g$ and $\operatorname{deg}_{y_{i 3}} f=\operatorname{deg}_{y_{i 31}} g$, the number of the components $f_{\ell}$ having $\left\{y_{i 1}, y_{i 3}, y_{i 4}\right\}$ are equal to the number of the components $g_{\ell}$ having $\left\{y_{i 1}, y_{i 3}, y_{i 4}\right\}$. Similarly the number of the components $f_{\ell}$ and $g_{\ell}$ which have $\left\{y_{i 2}, y_{i 4}, y_{i 5}\right\}$, and the number of the components $f_{\ell}$ and $g_{\ell}$ 
which have $\left\{y_{i 1}, y_{i 2}, y_{i 3}\right\}$ are equal. So the degree of the variables $y_{i 4}, y_{i 5}$ are equal in $f$ and $g$, a contradiction.

Case 6. $z=z_{i 1}$ or $z_{i 2}$ for some $i$ : Assume that the $z_{i 3}$ and $z_{i 4}$ be the other vertices in the 4-cycle corresponding to $L_{i}$. Since $z_{i 3}, z_{i 4} \in V\left(F_{1} \cup \cdots \cup F_{m} \cup G_{1} \cup \cdots \cup G_{k}\right)$, we have $z_{i 3}, z_{i 4}>_{\text {lex }} z_{i 1}>_{\text {lex }} z_{i 2}$. In every vertex cover $C$ of $G$ exactly one of the vertices $z_{i 1}, z_{i 2}$ appears. Moreover $C$ contains one of the subsets

$$
\left\{z_{i 1}, z_{i 3}\right\},\left\{z_{i 2}, z_{i 4}\right\},\left\{z_{i 1}, z_{i 3}, z_{i 4}\right\},\left\{z_{i 2}, z_{i 3}, z_{i 4}\right\} \text {. }
$$

Since $f$ and $g$ have the same degrees in the variables $z_{i 3}$ and $z_{i 4}$, the number of $f_{\ell}$ with $\left\{z_{i 1}, z_{i 3}\right\} \subset \operatorname{supp}\left(f_{\ell}\right)$ is equal to the number of $g_{\ell}$ with $\left\{z_{i 1}, z_{i 3}\right\} \subset \operatorname{supp}\left(g_{\ell}\right)$. Also the number of $f_{\ell}$ with $\left\{z_{i 2}, z_{i 4}\right\} \subset \operatorname{supp}\left(f_{\ell}\right)$ is equal to the number of $g_{\ell}$ with $\left\{z_{i 2}, z_{i 4}\right\} \subset$ $\operatorname{supp}\left(g_{\ell}\right)$. First assume that $z=z_{i 2}$. These two facts imply that the number of the $g_{\ell}$ with $\left\{z_{i 1}, z_{i 3}, z_{i 4}\right\} \subseteq \operatorname{supp}\left(g_{\ell}\right)$ is greater than the number of $f_{\ell}$ with this property and so $\operatorname{deg}_{z_{i 1}}(g)>\operatorname{deg}_{z_{i 1}}(f)$, a contradiction. So we have $z=z_{i 1}$ and the number of the $g_{l}$ 's with $\left\{z_{i 2}, z_{i 3}, z_{i 4}\right\} \subseteq \operatorname{supp}\left(g_{\ell}\right)$ is greater than the number of $f_{\ell}$ with this property. Now for some $g_{j}$ with $\left\{z_{i 2}, z_{i 3}, z_{i 4}\right\} \subseteq \operatorname{supp}\left(g_{j}\right)$, set $g_{j}^{\prime}=g_{j} z_{i 1} / z_{i 2}$. Since $N_{G}\left(z_{i 2}\right)=\left\{z_{i 1}, z_{i 3}\right\} \subset \operatorname{supp}\left(g_{j}\right)$ we have $\operatorname{supp}\left(g_{j}^{\prime}\right)$ is a vertex cover of $G$.

Example 3.4. The cactus graph given in Example 2.4 is Cohen-Macaulay by Theorem 3.3. With respect to the term order given in the proof of Theorem 2.3 we have that $J(G)^{k}$ is weakly polymatroidal ideals for each $k$.

\section{REFERENCES}

[1] A. Conca, J. Herzog, T. Hibi, Rigid resolutions and big Betti numbers, Comment. Math. Helv. 79 (2004) 826-839.

[2] J. Eagon, V. Reiner, Resolutions of Stanley-Reisner rings and Alexander duality, J. Pure Appl. Algebra 130 (1998) 265-275.

[3] S. Faridi, Simplicial trees are sequentially Cohen-Macaulay, J. Pure Appl. Algebra 190 (2004) 121-136.

[4] C. A. Francisco, H. T. Hà, Whiskers and sequentially Cohen-Macaulay graphs, J. Combin. Theory Ser. A. 115 (2008) 304-316.

[5] C. Francisco, A. Van Tuyl, Sequentially Cohen-Macaulay edge ideals, Proc. Amer. Math. Soc. 135 (2007) 2327-2337.

[6] J. Herzog, T. Hibi, Monomial ideals, Graduate Texts in Mathematics 260, Springer (2010).

[7] J. Herzog, T. Hibi, Distributive lattices, bipartite graphs, and Alexander duality, J. Algebraic Comb. 22 (2005) 289-302.

[8] J. Herzog, T. Hibi, The depth of powers of an ideal, J. Algebra 291 (2005) 534-550.

[9] J. Herzog, T. Hibi, Componentwise linear ideals, Nagoya Math. J. 153 (1999) 141-153.

[10] J. Herzog, T. Hibi, H. Ohsugi, Powers of componentwise linear ideals, Combinatorial aspects of commutative algebra and algebraic geometry, 49-60, Abel Symp., 6, Springer, Berlin, 2011.

[11] J. Herzog, T. Hibi, X. Zheng, Cohen-Macaulay chordal graphs, J. Combin. Theory Ser. A. 113 (2006) 911-916.

[12] J. Herzog, Y. Takayama, Resolutions by mapping cones, The Roos Festschrift volume, 2. Homology Homotopy Appl. 4 (2002) 277-294.

[13] M. Kokubo, T. Hibi, Weakly polymatroidal ideals, Algebra Colloq. 13 (2006) 711-720.

[14] F. Mohammadi, Powers of the vertex cover ideal of a chordal graph, Comm. in Algebra 39 (2011) 1-12.

[15] F. Mohammadi, S. Moradi, Weakly polymatroidal ideals with applications to vertex cover ideals, Osaka J. Math. 47 (2010) 627-636.

[16] F. Mohammadi, D. Kiani, S. Yassemi, Shellable cactus graphs, Math. Scand. 106 (2010) 161-167.

[17] E. Nero, Regularity of edge ideals of $C_{4}$-free graphs via the topology of the lcm-lattice, J. Combin. Theory Ser. A 118 (2011) 491-501.

[18] E. Nevo, I. Peeva, $C_{4}$-free edge ideals, J. Algebraic Combin. (2012). 
[19] R. P. Stanley, Combinatorics and Commutative Algebra. Second edition. Progress in Mathematics 41. Birkhäuser Boston, Inc., Boston, MA, (1996).

[20] B. Sturmfels, Four counterexamples in combinatorial algebraic geometry, J. Algebra 230 (2000) 282294.

[21] A. Van Tuyl, Sequentially Cohen-Macaulay bipartite graphs: vertex decomposability and regularity, Arch. Math. (Basel) (2009) 451-459.

[22] R. H. Villarreal, Cohen-Macaulay graphs, Manuscripta Math. 66 (1990) 277-293.

E-mail address: fatemeh.mohammadi716@gmail.com

Fachbereich Mathematik und Informatik, Philipps-Universität, 35032 Marburg, Germany 